\title{
EFEITOS DO TREINAMENTO INTERVALADO NA RESPOSTA HIPOTENSIVA DE MILITARES COM DIFERENTES PADRÕES DE CONDICIONAMENTO FÍSICO
}

\author{
MS. MATHEUS CAVALCANTE DE SÁ \\ Programa de Pós-graduação em Medicina (Otorrinolaringologia), Departamento de \\ Otorrinolaringologia e Cirurgia de Cabeça e Pescoço, Universidade Federal de São Paulo; \\ Centro de Preparação de Oficiais da Reserva de São Paulo, Exército Brasileiro \\ (São Paulo - São Paulo - Brasil) \\ Email:matssa@msn.com
}

\section{GRAD. EUDIMACI BARBOZA DE LIRA}

Escola de Educação Física do Exército, Exército Brasileiro

(Rio de Janeiro - Rio de Janeiro - Brasil)

Email: enomislira08@yahoo.com.br

\section{DR. ANTÔNIO FERNANDO ARAÚJO DUARTE \\ Divisão de Pesquisa, Instituto de Pesquisa e Capacitação Física de Exército, \\ Exército Brasileiro (Rio de Janeiro - Rio de Janeiro - Brasil) \\ Email: afduarte@globo.com}

\begin{abstract}
RESUMO
O exercício tem sido considerado um importante instrumento não farmacológico de controle da pressão arterial (PA). Nesse sentido, o presente estudo investigou os efeitos de uma sessão de alta intensidade de Treinamento Intervalado Aeróbio de corrida (TIA) sobre o nivel de Hipotensão Pós-esforço (HPE) de indivíduos com diferentes padrões de condiçãa fisica. Dezesseis homens jovens foram divididos em dois grupos: um com condicionamento aeróbio superior (GSup; $n=8 ; V O_{2 \max }=55,5 \mathrm{ml} \cdot \mathrm{kg}^{-1} \cdot \mathrm{min}^{-1}$ ) e outro inferior (Glnf; $n=8$; $\left.\mathrm{VO}_{2 \max }=42,1 \mathrm{ml} \cdot \mathrm{kg}^{-1} \cdot \mathrm{min}^{-1}\right)$. Os grupos realizaram sete repetições de $400 \mathrm{~m}$ de corrida, com intensidades de $117 \%$ (Glnf) e $113 \%$ (GSup) da velocidade relativa ao consumo máximo de oxigênio $\left(\mathrm{VVO}_{2 \text { max }}\right)$, com intervalos recuperadores de I min30s. Os valores de PA sistólica (PAS) e diastólica (PAD) foram registrados antes do esforço (Pré) e 0, 10, 20, 30, 45 e 60 min pós-esforço (Pós). Apenas o Glnf apresentou HPE na PAS no momento 60 min Pós em relação a Pré (I | 3,8 $\pm 2,5$ vs. 124,6 $22,2 \mathrm{mmHg}$; respectivamente, $p=0,03)$. Em relação à PAD, os dois grupos apresentaram HPE nas medidas Pós, comparativamente com o valor Pré - GInf a partir de 20 min e GSup de 10 min Pós $(p<0,02)$. O GInf apresentou PA média (PAM) Pós inferior a Pré durante os $60 \mathrm{~min}(p<0,01)$ e o GSup somente no momento 45 min ( $p<0,02)$. Não houve diferenças entre os grupos quanto à PAS, PAD e PAM ( $p>0,05)$.
\end{abstract}


Os resultados sugerem que uma maior intensidade relativa do TIA pode levar a uma redução mais pronunciada nos níveis de PA até uma hora pós-exercício.

PALAVRAS-CHAVE: Pressão arterial; hipotensão pós-esforço; treinamento intervalado; normotenso.

\section{INTRODUÇÃO}

A hipertensão arterial (HA) é considerada um dos fatores de risco e um importante preditor de doenças e comorbidades cardiovasculares (CHOBANIAN et al., 2003). No Brasil, de acordo com as IV Diretrizes Brasileiras de Hipertensão Arterial (2002), aproximadamente $40 \%$ dos indivíduos acometidos de HA são levados a óbito, sendo um dos problemas de saúde pública de maior prevalência na população. Segundo Cunha et al. (2006), o tratamento da HA é realizado por meio de medicamentos e deve estar associado a uma mudança de estilo de vida, como alterações no padrão alimentar e prática regular de exercícios físicos.

Diversos estudos têm investigado os efeitos fisiológicos da prática de exercícios físicos sobre a saúde de indivíduos normotensos e hipertensos, de ambos os sexos e de diversas idades (PONTES JÚNIOR et al., 20 I0; LIMA et al. 2012; SÁ; VICTORINO; VAISBERG, 20I2). Entre esses efeitos está a hipotensão pós-esforço (HPE), que é definida na literatura como a redução aguda e subaguda dos níveis pressóricos do indivíduo após a realização de sessões de exercício dinâmico, de intensidades de moderada a alta, com durações que variam de alguns minutos a horas (PESCATELLO et al., 2004). Somente a partir da década de 1980 iniciou-se sistematicamente a investigação desse fenômeno, relacionando-o como efeito clínico relevante (PESCATELLO et al., 1991). Alguns estudos identificaram a HPE em normotensos, porém com menor magnitude quando comparados a hipertensos (FORJAZ et al., 2000; BERMUDES et al., 2003; DE SALLES et al., 2010). Em indivíduos jovens e normotensos, reduções médias de 8 a $10 \mathrm{mmHg}$ são observadas na pressão arterial sistólica (PAS) por algumas horas pós-exercício (MACDONALD, 2002).

No entanto, não é consensual na literatura a relação entre intensidade e a duração do exercício e a magnitude da HPE em normotensos (FORJAZ et al., 1998; MACDONALD et al., 2000; JONES et al., 2008; CASONATTO; POLITO, 2009). Em se tratando do parâmetro intensidade do exercício, estudos relatam diferentes efeitos agudos do exercício aeróbio na redução dos níveis pressóricos de seus praticantes, após uma única sessão de exercícios físicos (BROWLEY et al., 2003; KULICS; COLLINS; DICARLO, 1999; JONES et al., 2007; LEGRAMANTE et al., 2002; RONDON; BRUM, 2003; SENITKO; CHARKOUDIAN; HALLIWIL, 
2002). Jones et al. (2007), por exemplo, verificaram diferenças na HPE entre a prática de exercício intenso e moderado. Acompanhando os sujeitos 20 minutos após o estímulo, o grupo submetido a exercício intenso demonstrou redução da resistência periférica total e aumento da condutância vascular cutânea. Dessa forma, pode-se supor que a intensidade do exercício influencie de alguma forma o comportamento da HPE.

Um dos métodos de treinamento cardiopulmonar prescritos no Manual de Campanha de Treinamento Físico Militar do Exército (BRASIL, 2002) é o Treinamento Intervalado Aeróbico (TIA). Realizado com base em uma distância de $400 \mathrm{~m}$, alterna estímulos de exercício com intensidade média-forte, intercalados por intervalos de recuperação parcial. Além de propiciar adaptações orgânicas que visam levar o praticante a um melhor desempenho físico, a realização do TIA pode, paralelamente, trazer benefícios para a saúde do militar, entre eles um melhor controle agudo de seus níveis pressóricos, comportamento este ainda não comprovado na literatura.

$O$ controle da intensidade do TIA é baseado no resultado do indivíduo em um teste de corrida de 12 min (COOPER, 1968). À distância atingida no teste, é acrescido um valor fixo em metros para efeito de cálculo do novo tempo de estímulo de treinamento (que tem por base a distância de 400m). Por exemplo, se um indivíduo atingiu 2800m no teste de corrida, soma-se $400 \mathrm{~m}$ a esse valor, o que leva a um resultado de $3200 \mathrm{~m}$. Divide-se esse montante pela distância alvo de treinamento $(400 \mathrm{~m})$, gerando o valor oito. Para o cálculo do tempo de estímulo, divide-se o tempo de teste de 12 min por oito, o que dá o tempo de I min30s para cada estímulo de $400 \mathrm{~m}$ de distância, para o treinamento intervalado.

Entretanto, essa sobrecarga fixa de distância para o cálculo do tempo do estímulo leva a diferentes intensidades de treino, em função da condição aeróbia do praticante, que, por sua vez, guarda relação direta com seu resultado no teste de 12 min. Em função dos diversos estudos que trataram do efeito da intensidade do exercício sobre a HPE (BROWLEY et al., 2003; KULICS; COLLINS; DICARLO, 1999; JONES et al., 2007; LEGRAMANTE et al., 2002; RONDON; BRUM, 2003; SENITKO; CHARKOUDIAN; HALLIWIL, 2002), pode-se hipotetizar que a variação na intensidade do treinamento, fruto de uma sobrecarga fixa sobre indivíduos com diferentes padrões de condição aeróbia, pode levar a diferentes comportamentos do controle pressórico pós-esforço.

Assim, o presente estudo tem por finalidade avaliar os efeitos de uma sobrecarga fixa na intensidade do TIA, caracterizado pelo acréscimo de 400m ao resultado do teste de corrida de 12 min (COOPER, 1968) para o cálculo do tempo de estímulo, sobre o nível de HPE de militares com diferentes padrões de condicionamento aeróbio. 


\section{SUJEITOS}

Participaram do estudo 16 sujeitos fisicamente ativos, com histórico de realização de atividades aeróbias há pelo menos um ano, divididos em dois grupos de oito integrantes, de acordo com o nível de condicionamento físico. Todos foram voluntários, sendo militares da ativa e normotensos. $\bigcirc$ grupo de condicionamento superior (GSup; n=8) foi composto por sujeitos que atingiram a distância de $3000 \mathrm{~m}$ no teste inicial de corrida de 12 minutos (COOPER, 1968), e o grupo de condicionamento inferior ( $\mathrm{G}$ Inf; $n=8$ ), por militares que atingiram $2400 \mathrm{~m}$ no mesmo teste.

Todos os militares foram informados dos objetivos e implicações do estudo e assinaram, previamente, um termo de consentimento, no qual estavam explicitados todos os procedimentos a que seriam submetidos, de acordo com a resolução 196/96 do Conselho Nacional de Saúde. O projeto foi aprovado pelo Comitê de Ética em Pesquisa da Universidade Federal de São Paulo (CAAE: OI 952 I 2.0.0000.5505). As características dos participantes do estudo são apresentadas na Tabela I.

Tabela I. Características dos sujeitos (Média \pm EPM)

\begin{tabular}{lcccc}
\hline Variáveis & G-Sup & G-Inf & $\mathrm{t}(14)$ & Valor de P \\
\hline Idade (anos) & $21,7 \pm 2,1$ & $19,4 \pm 1,3$ & 2,76 & $0,02 *$ \\
Peso $(\mathrm{kg})$ & $72,9 \pm 10,2$ & $75,7 \pm 15,9$ & $-0,43$ & 0,67 \\
Altura $(\mathrm{cm})$ & $169,4 \pm 8,3$ & $174,2 \pm 7,9$ & $-1,18$ & 0,26 \\
IMC & $25,4 \pm 3,1$ & $24,9 \pm 4,4$ & 0,26 & 0,80 \\
\% Gordura & $13,6 \pm 3,7$ & $17,1 \pm 5,6$ & $-1,45$ & 0,17 \\
PAS $_{\text {Repouso }}(\mathrm{mmHg})$ & $117,6 \pm 10,1$ & $124,6 \pm 2,2$ & 0,86 & 0,41 \\
PAD $_{\text {Repouso }}(\mathrm{mmHg})$ & $76,0 \pm 6,7$ & $80,6 \pm 8,9$ & $-0,14$ & 0,89 \\
PAM $_{\text {Repouso }}(\mathrm{mmHg})$ & $89,0 \pm 1,7$ & $94,6 \pm 7,1$ & 0,23 & 0,82 \\
\hline
\end{tabular}

G-Sup, grupo de condicionamento físico superior; G-Inf, grupo de condicionamento físico inferior; $t$ - teste de amostra independente, $p$ - significância estatística, * Diferença estatisticamente significativa.

\section{PROCEDIMENTOS}

Para o início das avaliações, os participantes foram conduzidos ao laboratório, onde foram orientados sobre o propósito do estudo e assinaram o termo de consentimento livre e esclarecido. Nesta mesma visita, foram realizadas as mensurações de peso, altura, percentual de gordura (\% Gordura), PAS e pressão arterial 
diastólica (PAD) de repouso $\left(\mathrm{PAS}_{\text {Repouso }} ; \mathrm{PAD}_{\text {Repouso }}\right)$ para caracterização da amostra estudada. $O$ percentual de gordura foi avaliado pelo protocolo de sete dobras cutâneas (JACKSON; POLLOCK, 1978). Após 10 minutos em repouso, com os sujeitos deitados na posição supina, a PAS e PAD foram mensuradas pelo método auscultatório, por um avaliador treinado. A pressão arterial média (PAM) foi calculada como sendo a soma da PAS com duas vezes o valor de PAD, tudo dividido por três. Em uma segunda etapa, a amostra foi submetida a um teste de corrida de $12 \mathrm{~min}$ (COOPER, 1968) em uma pista de atletismo de 400m.

Em uma visita subsequente, os participantes foram submetidos a uma sessão de TIA composta por sete repetições de 400m de distância. $O$ cálculo da intensidade de cada estímulo foi feito da maneira descrita a seguir, de acordo com o que prescreve o Manual de Campanha de Treinamento Físico Militar do Exército (BRASIL, 2002): (a) foi feito um acréscimo de 400m à distância atingida no teste de I 2 min por cada grupo; (b) dividiu-se o valor total obtido em (a) pela distância alvo de treinamento (400m); (c) dividiu-se, então, o tempo de 12 min pelo resultado de (b) para encontrar o tempo de estímulo para cada grupo. Todas as avaliações foram realizadas por volta das 08:00 horas da manhã, a uma temperatura média de $23^{\circ} \mathrm{C}$ e umidade relativa do ar aproximada de $72 \%$.

Antes das sessões de TIA e após um período inicial de 5 min na posição supina (Pré), os sujeitos tiveram as variáveis PAS e PAD de repouso auferidas por meio do monitor Multiparâmetros INMAX (Instramed ${ }^{\circledR}$, Porto Alegre, Brasil). Para fins de controle, durante os intervalos recuperadores ao longo da sessão de TIA, os valores de PA foram registrados por um avaliador experiente, pelo método auscultatório, com o indivíduo na posição ortostática. A PA de esforço (Esf) foi medida imediatamente após a realização da última repetição, com o indivíduo na posição supina, por meio do monitor Multiparâmetros INMAX (Instramed ${ }^{\circledR}$, Porto Alegre, Brasil).

Para a verificação dos níveis de HPE, ao término da sessão de TIA, os sujeitos foram conduzidos para uma sala de avaliação, colocados na posição supina, e tiveram seus valores de PAS e PAD registrados nos tempos de 10 min, 20 min, 30 min, 45 min e 60 min pós-teste, por meio do aparelho Multiparâmetros INMAX (Instramed $^{\circledR}$, Porto Alegre, Brasil).

\section{ANÁLISE ESTATIISTICA}

Após a análise da normalidade da distribuição dos dados, pelo teste de Shapiro-Wilk, as características dos grupos foram comparadas por meio de testes- $t$ para amostras independentes. A comparação entre as medidas de PA realizadas em laboratório e no campo, pelos métodos auscultatório e automatizado, respectivamente, 
foi realizada por teste-t para amostras dependentes, considerando-se as medidas de todos os participantes de maneira conjunta, independentemente dos grupos. A análise do comportamento de cada variável, PAS, PAD e PAM, ao longo dos momentos de avaliação e entre os grupos GSup e GInf, foi realizada por meio do teste ANOVA fatorial com medidas repetidas, seguida pelo post-hoc de Tukey. O nível de significância estatística adotado foi de $p<0,05$. Para a realização dos cálculos estatísticos foi utilizado o software Statistica 7.I (StatSoft, EUA).

\section{RESULTADOS}

Para a realização do estudo, o GSup e o Glnf foram constituídos por oito participantes cada. Embora os grupos não contassem com um número elevado de sujeitos, a potência estatística dos testes empregados não foi afetada. Levando-se em conta as variáveis analisadas e o tamanho amostral, a menor potência estatística observada foi de 0,99.

No teste inicial de 12 min, os integrantes do GSup atingiram a distância de 3000m e os do Glnf, 2400m. Por meio do cálculo do consumo máximo de oxigênio proposto por Cooper ( 1968 ), os valores estimados de $\mathrm{VO}_{2 \max }$ foram de $55,5 \mathrm{ml} \cdot \mathrm{kg}^{-1} \cdot \mathrm{min}^{-1}$ para o GSup e de $42, \mathrm{I} \mathrm{ml} \cdot \mathrm{kg}^{-1} \cdot \mathrm{min}^{-1}$ para o $\mathrm{G} \mathrm{Inf}$. Esse resultado se traduziu em intensidades de estímulo de 1 17\% da velocidade relativa ao consumo máximo de oxigênio $\left(\vee V O_{2 \max }\right)$ para o Glnf e I $13 \%$ da $\vee \mathrm{O}_{2 \max }$ para o GSup. Considerou-se a $\mathrm{VO}_{2 \max }$ como a velocidade média desenvolvida no teste máximo de corrida de 12 min (HILL; ROWELL, 1997).

A comparação das medidas de repouso de PAS e PAD realizadas no laboratório, pelo método auscultatório, e no campo, pelo método automatizado, não apresentou diferenças estatísticas. O comportamento da PAS foi de 1 19,7 \pm 10, I $\mathrm{mmHg}$ no laboratório e de $121,9 \pm 5,9 \mathrm{mmHg}$ no campo $(p=0,16)$. A PAD igualmente não apresentou diferenças entre as medidas auscultatória e automatizada (78,3 $\pm 8,7$ vs. $80,0 \pm 6,7 \mathrm{mmHg}$, respectivamente; $p=0,34)$.

Com relação à variável PAS (FIGURA I), não foi observado efeito hipotensivo nos integrantes do GSup. Nenhuma medida pós-esforço foi significativamente menor do que o valor inicial de PAS $(p>0,21$ ). Com relação ao GInf, somente na medida realizada 60 min após o TIA foi verificada HPE, comparativamente ao valor de repouso ( $124,6 \pm 2,2$ vs. | | 3,8 $\pm 2,5 \mathrm{mmHg}, p=0,03$ ). Apesar disso, não houve diferença significativa entre os grupos nos 60 minutos pós-exercício monitorados. 


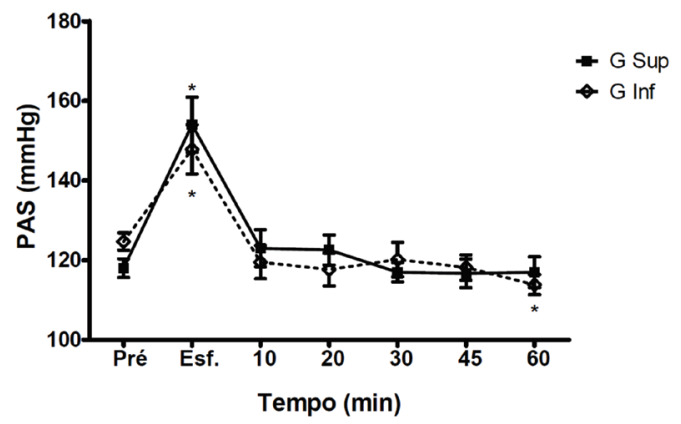

Figura I. Valores médios ( \pm EPM) da PAS inicial (Pré), imediatamente após a execução do TIA (Esf.) e nos intervalos de recuperação ( 10 min. a 60 min.), após a sessão de treinamento. (* $p=0,03$ em relação ao início do teste do teste).

A análise dos valores de PAD revelou um comportamento hipotensivo em ambos os grupos avaliados $(p<0,02)$ em comparação aos níveis de repouso. Os sujeitos de melhor condicionamento aeróbio (GSup) apresentaram HPE a partir de 10 min pós-esforço e os sujeitos do Glnf, a partir do minuto 20. Da mesma forma como a PAS, não foi observada diferença significativa entre os grupos no que se refere à PAD (FIGURA 2).

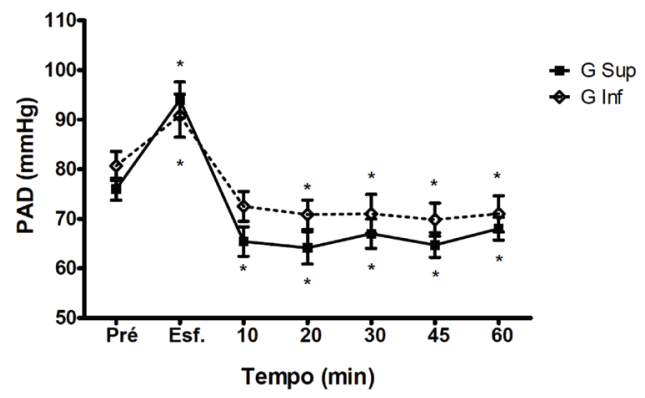

Figura 2. Valores médios ( \pm EPM) da PAD inicial (Pré), imediatamente após a execução do TIA (Esf.) e nos intervalos de recuperação ( 10 min a 60 min), após a sessão de treinamento. (* $p<0,02$ em relação ao início do teste).

Em relação ao comportamento da PAM, ambos os grupos apresentaram hipotensão em relação à medida de repouso, mas também não foi observada diferença significativa entre os grupos (FIGURA 3). No entanto, os sujeitos de menor condicionamento aeróbio (Glnf) apresentaram um comportamento hipotensivo em todo o período de recuperação ( $p<0,02)$. Nos sujeitos do GSup, foi observada HPE somente no minuto 45 , considerando-se a medida de repouso $(89,0 \pm 1,7$ vs $82,0 \pm 2,7 \mathrm{mmHg}, p=0,02)$. 


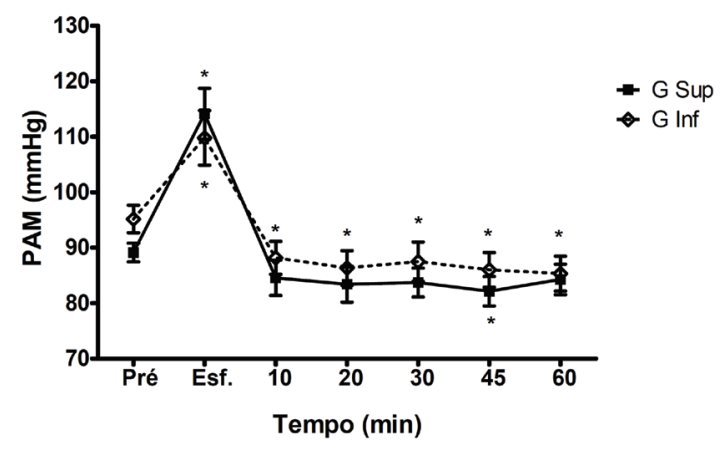

Figura 3. Valores médios ( \pm EPM) da PAM inicial (Pré), imediatamente após a execução do TIA (Esf.) e nos intervalos de recuperação ( 10 min a 60 min), após a sessão de treinamento. (* $p<0,02$ em relação ao início do teste).

\section{DISCUSSÃO}

O presente estudo procurou investigar a ocorrência de HPE em indivíduos com diferentes padrões de condicionamento aeróbio, por um período de até $60 \mathrm{~min}$ após uma sessão aguda de TIA, executado a uma velocidade acima de $100 \% \mathrm{VVO}_{2 \text { max' }}$ conforme sugerido pelo Manual de Treinamento Físico Militar do Exército (2002). Os resultados indicaram que uma sobrecarga fixa de intensidade no planejamento do TIA, pode levar a um maior efeito hipotensivo pós-esforço em indivíduos com menor padrão de condicionamento aeróbio, em comparação com indivíduos mais aptos, considerando-se as características dos grupos estudados.

No que diz respeito à PAS, foi evidenciada HPE nos indivíduos com condicionamento aeróbio inferior (Glnf) na medida realizada 60 min após o esforço, sendo que o mesmo não foi observado naqueles de condição superior (GSup). Alguns estudos (DUJIC et al., 2006; LIU et al., 20 I2) indicam que maiores valores de HPE estariam associados a maiores valores de PAS medida em repouso. DUJIC et al.(2006) submeteram indivíduos fisicamente ativos e normotensos a uma sessão de corrida a $100 \%$ do $\mathrm{VO}_{2 \text { max }}$, em uma pista de atletismo, e relataram uma diminuição dos níveis pressóricos durante todo o período pós-esforço monitorado (60 min), sendo maior o efeito hipotensivo nos possuidores de níveis mais elevados de PAS de repouso. No presente estudo, embora o Glnf tenha apresentado um valor médio de PAS no pré-teste (Pré) ligeiramente mais alto do que o GSup, a pequena diferença observada entre os grupos não foi estatisticamente significativa, indicando que a ocorrência do efeito hipotensivo na PAS do GInf pode ser derivada, entre outras causas, da redução da resistência periférica total devido aos efeitos de uma 
maior intensidade relativa do exercício para seus integrantes em relação aos do GSup. Na investigação de Liu et al. (20।2), além de uma maior PAS de repouso estar relacionada a uma maior HPE, maiores intensidades de esforço levaram a uma maior redução pressórica pós-esforço.

Alguns estudos (CORNELISSEN; FAGARD, 2004; FORJAZ et al., 2004) realizados com sujeitos jovens e normotensos relatam que exercícios aeróbicos dinâmicos, com durações variando de 20 a 45 min e intensidades de 50\% a 80\% do $\mathrm{VO}_{2 \text { max' }}$, podem não interferir na magnitude do efeito hipotensivo pós-esforço quanto à PAD. Esse mecanismo foi descrito por Halliwill et al. (20I2) e pode ser consequência da baixa intensidade de esforço que tende a não provocar a combinação de fatores importantes para a manifestação da HPE, como a diminuição na atividade nervosa simpática e a ação de mecanismos vasodilatadores locais. Por outro lado, no presente estudo, um nível maior de intensidade ( I 13\% e 1 17\% da $\checkmark \mathrm{VO}_{2 \text { max }}$ ) de esforço, mesmo de curta duração, associado à boa condição de saúde dos participantes (WISLOFF; ELLINGSEN; KEMI, 2009) pode ter sido a responsável pela hipotensão observada na PAD.

Em relação à PAM, embora a sessão de TIA não tenha sido longa e não tenha havido diferenças entre os grupos, é possível que a intensidade relativamente mais alta a que foi submetido o GInf tenha proporcionado o efeito de HPE durante os 60 min pós-esforço monitorados, o que não foi observado no GSup. Não existe, contudo, consenso na literatura em relação à intensidade do exercício aeróbio e sua magnitude e duração da HPE (CASONATTO; POLITO, 2009). A maioria dos estudos utilizaram intensidades que variaram de 40 a 100\% da capacidade máxima, medida por meio do $\mathrm{VO}_{2 \text { max }}$, frequência cardíaca de reserva ( $\left.\mathrm{FC}_{\text {res }}\right)$ ou frequência cardíaca máxima $\left(F C_{\text {max }}\right)$, e concluíram que a HPE acontece, em geral, até 60 min após a sessão de treinamento (BROWNLEY et al., 1996; HALLIWILL et al., 1996; HALLIWILL et al., 2000; LOCKWOOD et al., 2005; SHARMAN et al., 2008). Por outro lado, uma recente investigação conduzida por Cosonato et al. (20 I I) procurou comparar as respostas cardiovasculares e autonômicas após sessões de treino com diferentes intensidades e durações e não observou efeito hipotensivo em nenhum dos grupos avaliados, independentemente dos variados parâmetros de treino empregados.

\section{CONCLUSÕES}

Uma sobrecarga fixa na intensidade do TIA, levando a diferentes níveis de esforço de treinamento para militares com performances de $2400 \mathrm{~m}$ e $3000 \mathrm{~m}$ no teste de 12 min, não promoveu, nesses indivíduos, HPE de diferente magnitude, até 60 min pós-esforço. 
Considerando que a magnitude e a duração do efeito hipotensivo pós-esforço depende da relação entre o tempo e a intensidade do exercício, o volume do TIA de $400 \mathrm{~m}$ de elevada intensidade, realizado a sete repetições, não foi suficiente para promover efeito hipotensivo subagudo constante na PAS e PAM de indivíduos com melhor condição aeróbia. Ao contrário, indivíduos de menor condição aeróbia podem se beneficiar mais da redução dos níveis pressóricos, mantida por até uma hora pós-exercício, por ocasião da realização de protocolos de TIA semelhantes.

Finalmente, estudos adicionais podem ser realizados empregando o modelo de TIA constante do Manual de Treinamento Físico Militar, C 20-20 (BRASIL, 2002), com diferentes intensidades e tempos de estímulo e recuperação, a fim de investigar possíveis variações nas respostas hipotensivas apresentadas em função da aplicação de diferentes cargas de treinamento.

\section{Effects of Aerobic Running Interval Training in Hypotensive Response of Military with Different Standards of Aerobic Conditions}

ABSTRACT: Exercise has been considered an important non-pharmacological intervention to control blood pressure (BP). Therefore, the present study investigated the effects of one session of high intensity Aerobic Interval Training (AIT) upon post-exercise hypotension (PEH) levels in subjects with different fitness patterns. Sixteen young men were separated in two groups: one with higher $\left(\mathrm{GH} ; n=8 ; V O_{2 \max }=55.5 \mathrm{ml} \cdot \mathrm{kg}^{-1} \cdot \mathrm{min}^{-1}\right)$ and the other with lower ( $\mathrm{GL} ; n=8$; $\left.V_{2 \max }=42.1 \mathrm{ml} \cdot \mathrm{kg}^{-1} \cdot \mathrm{min}^{-1}\right)$ aerobic conditioning. Both groups performed seven repetitions of $400 \mathrm{~m}$ running, with intensities of $117 \%(G L)$ and $113 \%(G H)$ of the velocity at maximal oxygen uptake $\left(\mathrm{VVO}_{2 \text { max }}\right)$, with I min30s recovery intervals between stimuli. Systolic (SBP) and diastolic (DBP) BP values were registered before (Pre) and at 0, 10, 20, 30, 45 and $60 \mathrm{~min}$ post-exercise (Post). Only GL showed PEH at $60 \mathrm{~min}$ in Post compared to Pre (I I 3.8 22.5 vs. 124.6 $\pm 2.2 \mathrm{mmHg}$; respectively, $p=0.03)$. In regard to $D B P$, both groups presented $P E H$ in Post compared to Pre - GL from 20 min and GH from 10 min until 60 min Post $(p<0.02)$. $G L$ showed a lower mean BP (MBP) in Post compared to Pre during the 60 min registered $(p<0.01)$ and $\mathrm{GH}$ only at minute $45(p<0.02)$. There were no differences between groups in regard to SBP, DBP and $M B P(p>0.05)$. The results suggest that higher relative intensities of AlT may induce to a more pronounced reduction in BP levels until one hour after the effort. KEYWORDS: Blood Pressure; Post-exercise Hypotension; Interval Training; Normotensive.

\section{Efectos del entrenamiento de intervalos aeróbico de correr en la respuesta hipotensora de los militares con diferentes estándares de condiciones aeróbicas.}

RESUMEN: El ejercicio se ha considerado una importante intervención no farmacológica para controlar la presión arterial (PA). En consecuencia, el presente estudio investigó los efectos de una sesión de Entrenamiento de Intervalo Aeróbica de alta intensidad (EIA) en los niveles 
de Hipotensión Pos-Ejercicio (HPE) en sujetos con diferentes patrones de acondicionamiento aeróbico. Dieciséis hombres jóvenes fueron separados en dos grupos: uno con una mayor (GSup, $n=8$; VO2 máx $=55.5 \mathrm{ml} \bullet \mathrm{kg}$ - $1 \bullet$ min- ) y el otro con inferiores (Glnf; $n=8$; VO2max $=42.1 \mathrm{ml} \bullet \mathrm{kg}-1 \cdot \mathrm{min}-\mathrm{I}$ ) acondicionamientos aeróbicos. Ambos grupos realizaron siete repeticiones de $400 \mathrm{~m}$ de corrida, con intensidades de 11 \%\% (Glnf) y 1 13\% (GSup) de la velocidad en el consumo máximo de oxígeno (VVO2máx), con intervalos de recuperación de I min30s entre estímulos. Valores de PA Sistólica (PAS) y Diastólica (PAD) se registraron antes (Pre) y 0, 10, 20, 30, 45 y 60 min después del ejercicio (Pos). Sólo Glnf demostró HPE a 60 min del anuncio en comparación con Pre (I 13,8 $\pm 2,5$ vs 124,6 $\pm 2,2 \mathrm{mmHg}$, respectivamente, $p=0,03)$. En lo que respecta a la $P A D$, ambos grupos presentaron HPE en lo Pos cuando comparados con el pre - GInf de 20 min y la GSup de 10 min hasta 60 min después ( $p<0,02)$. Glnf mostró una menor media de PA (PAM) en Pos comparando-se con el pre durante los 60 min registrado ( $p<0,01$ ), y GSup sólo en 45 minutos $(p<0,02)$. No hubo diferencias entre los grupos en lo que respecta a la PAS, PAD y PAM $(p>0,05)$. Los resultados sugieren que las intensidades relativas más altas de EIA pueden conducir a una reducción más acentuada en los niveles de PA hasta una hora después del esfuerzo.

PALABRAS CLAVE: Presión arterial; hipotensión arterial; entrenamiento de intervalos; normotensos.

\section{REFERÊNCIAS}

BERMUDES, A. M. et al. Ambulatory blood pressure monitoring in normotensive individuals undergoing two single exercise sessions: resistive exercise training and aerobic exercise training. Arquivos Brasileiros de Cardiologia, São Paulo, v. 8I, n. I, p. 65-7I, jan. 2003.

BRASIL. Ministério da Defesa. Exército Brasileiro. Estado-Maior do Exército. Manual de campanha: treinamento físico militar (C 20-20). 3. ed. Brasília: EGGCF, 2002.

BROWNLEY, K. A. et al. Acute aerobic exercise reduces ambulatory blood pressure in borderline hypertensive men and women. American Journal of Hypertension, Nova York, v. 9, n. 3, p. 200-206, mar. 1996.

BROWNLEY, K. A. et al. Sympathoadrenergic mechanisms in reduced hemodynamic stress responses after exercise. Medicine and Science in Sports and Exercise, Hagerstown, v. 35, n. 6, p. 978-986, june 2003

CASONATTO, J. et al. Cardiovascular and autonomic responses after exercise sessions with different intensities and durations. Clinics, São Paulo, v. 66, n. 3, p. 453-458, 201 I.

CASONATTO, J; POLITO, M. D. Hipotensão pós-exercício aeróbio: uma revisão sistemática. Revista Brasileira de Medicina do Esporte, São Paulo, v. 15, n. 2, p. I 5 | - I 57, 2009.

CHOBANIAN, A. V. et al. The Seventh Report of the Joint National Committee on Prevention, Detection, Evaluation, and Treatment of High Blood Pressure: the JNC 7 report. JAMA, Chicago, v. 289, n. 19, p. 2560-2572, may 2003. 
COOPER, K. H. Testing and developing cardiovascular fitness within the United States Air Force. Journal of Occupational Medicine, Filadélfia, v. I0, n. II, p. 636-639, nov. 1968.

CORNELISSEN, V. A.; FAGARD, R. H. Exercise intensity and postexercise hypotension. Journal of Hypertension, Londres, v. 22, n. 10, p. I859- I86।, oct. 2004.

CUNHA, G. A. D. et al. Hipotensão pós-exercício em hipertensos submetidos ao exercício aeróbio de intensidades variadas e exercício de intensidade constante. Revista Brasileira de Medicina do Esporte, São Paulo, v. 12, n. 6, p. 313-317, 2006.

DE SALLES, B. F. et al. Influence of rest interval lengths on hypotensive response after strength training sessions performed by older men. Journal of Strength and Conditioning Research, Colorado Springs, v. 24, n. II , p. 3049-3054, nov. 2010.

DUJIC, Z. et al. Postexercise hypotension in moderately trained athletes after maximal exercise. Medicine and Science in Sports and Exercise, Hagerstown, v. 38, n. 2, p. 318-322, feb. 2006.

FORJAZ, C. L. et al. Postexercise hypotension and hemodynamics: the role of exercise intensity. Journal of Sports Medicine and Physical Fitness, Torino, v. 44, n. I, p. 54-62, mar. 2004.

FORJAZ, C. L. et al. Effect of exercise duration on the magnitude and duration of post-exercise hypotension. Arquivos Brasileiros de Cardiologia, São Paulo, v. 70, n. 2, p. 99-104, fev. 1998.

FORJAZ, C. L. et al. Factors affecting post-exercise hypotension in normotensive and hypertensive humans. Blood Pressure Monitoring, Londres, v. 5, n. 5/6, p. 255-262, oct./dec. 2000.

HALLIWILL, J. R. et al. Augmented baroreflex heart rate gain after moderate-intensity, dynamic exercise. American Journal of Physiology, Bethesda, v. 270, n. 2, pt. 2, p. R420-R426, feb. 1996.

HALLIWILL, J. R.; MINSON, C. T.; JOYNER, M. J. Effect of systemic nitric oxide synthase inhibition on postexercise hypotension in humans. Journal of Applied Physiology, Bethesda, v. 89, n. 5, p. 1830-1836, nov. 2000.

HALLIWILL, J. et al. Post-exercise hypotension and sustained post-exercise vasodilation: What happens after we exercise? Experimental Physiology, Dublin, 2012. Disponível em: <http: www.ep.physoc.org>. Acesso em: 28 maio 2012.

HILL, D. W.; ROWELL, A. L. Responses to exercise at the velocity associated with $\mathrm{VO}_{2 \max }$. Medicine and Science in Sports and Exercise, Hagerstown, v. 29, n. I, p. I 13-1 16, jan. 1997.

JACKSON, A. S.; POLLOCK, M. L. Generalized equations for predicting body density of men. British Journal of Nutrition, Cambridge, v. 40, n. 3, p. 497-504, nov. 1978.

JONES, $\mathrm{H}$. et al. Is the magnitude of acute post-exercise hypotension mediated by exercise intensity or total work done? European Journal of Applied Physiology, Heidelberg, v. I02, n. I, p. 33-40, dec. 2007. 
JONES, $\mathrm{H}$. et al. The acute post-exercise response of blood pressure varies with time of day. European Journal of Applied Physiology, Heidelberg, v. I04, n. 3, p. 48I -489, oct. 2008.

KULICS, J. M.; COLLINS, H. L.; DICARLO, S. E. Postexercise hypotension is mediated by reductions in sympathetic nerve activity. American Journal of Physiology, Bethesda, v. 276, n. I, p. 27-32, Jan. 1999.

LEGRAMANTE, J. M. et al. Hemodynamic and autonomic correlates of postexercise hypotension in patients with mild hypertension. American Journal of Physiology: regulatory, integrative and comparative physiology, Bethesda, v. 282, n. 4, p. 1037-1043, apr. 2002.

LIMA, R. M. et al. Estudo de associação entre força muscular e massa magra em mulheres idosas. Revista Brasileira de Ciências do Esporte, Porto Alegre, v. 34, n. 4, dez. 2012.

LIU, S. et al. Blood pressure reduction following prolonged exercise in young and middleaged endurance athletes. European Journal of Preventive Cardiology, Thousand Oaks, july 2012. Disponível em: <http://cpr.sagepub.com/>. Acesso em: 10 jul. 2012.

LOCKWOOD, J. M. et al. Postexercise hypotension is not explained by a prostaglandindependent peripheral vasodilation. Journal of Applied Physiology, Bethesda, v. 98, n. 2, p. 447-453, feb. 2005.

MACDONALD, J. R. Potential causes, mechanisms, and implications of post exercise hypotension. Journal of Human Hypertension, Birmingham, v. I6, n. 4, p. 225-236, apr. 2002.

MACDONALD, J. R.; MACDOUGALL, J. D.; HOGBEN, C. D. The effects of exercise duration on post-exercise hypotension. Journal of Human Hypertension, Birmingham, v. I4, n. 2, p. 125-1229, feb. 2000.

PESCATELLO, L. S. et al. Short-term effect of dynamic exercise on arterial blood pressure. Circulation, Dallas, v. 83, n. 5, p. I557-|56|, may 199|.

PESCATELLO, L. S. et al. American College of Sports Medicine position stand. Exercise and hypertension. Medicine and Science in Sports and Exercise, Hagerstown, v. 36, n. 3, p. 533-553, mar. 2004.

PONTES JUNIOR, F. L. et al. Influência do treinamento aeróbio nos mecanismos fisiopatológicos da hipertensão arterial sistêmica. Revista Brasileira de Ciências do Esporte, Porto Alegre, v. 32, n. 2/4, dez. 2010.

RONDON, M. U. P. B.; BRUM, P. C. Exercício físico como tratamento não-farmacológico da hipertensão arterial. Revista Brasileira de Hipertensão, Ribeirão Preto, v. I0, n. 2, p. I34139, abr.jun. 2003.

SÁ, M. C.; VICTORINO, A. B.; VAISBERG, M. W. Incidência de lesão musculoesquelética sem trauma em atletas de handebol. Revista Brasileira de Medicina do Esporte, São Paulo, v. 18, n. 6 , dez. 2012 
SENITKO, A.; CHARKOUDIAN, N.; HALLIWILL, J. R. Influence of endurance exercise training status and gender on postexercise hypotension. Journal of Applied Physiology, Bethesda, v. 92, n. 6, p. 2368-2374, jun. 2002.

SHARMAN, J. E. et al. Nitric oxide does not significantly contribute to changes in pulse pressure amplification during light aerobic exercise. Hypertension, Dallas, v. 5I, n. 4, p. 856-61, apr. 2008.

SOCIEDADE BRASILEIRA DE HIPERTENSÃO ARTERIAL. IV Diretrizes Brasileiras de Hipertensão Arterial. São Paulo, 2002.

WISLOFF, U.; ELLINGSEN, O.; KEMI, O. J. High-intensity interval training to maximize cardiac benefits of exercise training? Exercise Sport Science Review, Filadélfia, v. 37, n. 3, p. 139- | 46, july 2009.

Recebido em: 2 mai. 2012 Aprovado em: 11 jun. 2013 Endereço para correspondência: Antônio Fernando Araújo Duarte Instituto de Pesquisa da Capacitação Física do Exército Av João Luis Alves, S/N Fortaleza de São João, Urca Rio de Janeiro-RJ CEP: 22.291-090 